\title{
Una aproximación semiótica empírica a la comprensión lectora*
}

\author{
A semiotic empirical approximation to reading comprehension
}

\author{
J orge Lagos, ${ }^{a}$ Ana M aría Soza, Sebastián Zepeda, Daniel Rojas \\ aUniversidad de Tarapacá, Departamento de Español, Casilla 6-D, Arica, Chile. Correo electrónico: \\ jlagos@uta.cl
}

El presente trabajo describe y analiza semióticamente, en el marco de los niveles de cooperación textual de Umberto Eco, la actualización del contenido de un ensayo literario realizada por estudiantes de ingreso 2010 a las carreras de Licenciatura de la Facultad de Educación y Humanidades de la Universidad de Tarapacá de Arica.

Palabras clave: semiótica, lector modelo, lector empírico, estructuras de mundo, paseos inferenciales.

The present work semiotically describes and analyzes, within the framework of Umberto Eco's textual cooperation levels, the "actualization" of a literary essay content realized by pupils (admission 2010) who are doing degree courses in the School of Education and Humanities at Universidad de Tarapacá.

Key words: Semiotic, model reader, empirical reader, world structures, inference making.

\section{INTRODUCCIÓN}

Es innegable en la actualidad la preocupación en todos los estamentos educacionales por los niveles alcanzados por los estudiantes en comprensión de lectura.

Hasta ahora el tema ha sido tratado fundamentalmente desde un enfoque disciplinario lingüístico, tanto en los diagnósticos realizados como en los avances posteriores de propuestas para sus eventuales mejorías. Otras orientaciones para abordar la comprensión lectora han sido las teorías discursivas, los enfoques

\footnotetext{
Este trabajo es parte de los resultados del Proyecto de Investigación Mayor Ciencia y Tecnología No 5740-10 de la Universidad de Tarapacá de Arica y fue presentado como ponencia en el XVIII Congreso Internacional de la Sociedad Chilena de Estudios Literarios (SOCHEL), realizado del 7 al 10 de noviembre de 2012 en la Universidad de Los Lagos, Osorno, Chile. Asimismo, la "lectura modelo" aludida en este artículo y que constituye punto de referencia de lectura de los resultados aquí dados se encuentra en Lagos y Rojas (2012). Se agradece el apoyo del Convenio de Desempeño Universidad de Tarapacá-Mineduc.
} 
multidisciplinarios de la ciencia cognitiva, la lingüística del texto, de la pragmática, de la socio y psicolingüística. Estudios que mayoritariamente se orientan a la identificación de elementos semánticos y sintácticos, elementos macro y microestructurales (Van Dijk 1995, 1996, 2001) o al desarrollo de estrategias y competencias del lector (Peronard 2005, 2007).

La concepción de la lectura y del buen lector, activo y participativo, de tales investigaciones tiene que ver, en general, con la selección adecuada de estrategias acordes a los objetivos de lectura, como se observa en estudios de Peronard, Gómez, Parodi y Núñez (1997) que definen al lector experto como aquel que posee un mayor desarrollo metacomprensivo en el marco de la sala de clases.

Por ello, al iniciar el estudio, constatamos que no ha habido intentos investigativos empíricos desde enfoques semióticos que permitan visualizar el fenómeno de la lectura dentro de un espectro más amplio que incluya implícitamente otras variables que influyen en ese proceso, como por ejemplo, y en primer lugar, la revisión del concepto de ‘comprensión lectora’ mismo, los niveles socioculturales de los lectores, las tipologías textuales en relación a la "enciclopedia” (Eco1981) de los lectores, etc. En otros términos, y globalmente, la consideración societal y cultural del proceso que cada vez está más presente en nuestro medio educacional.

En este sentido, este trabajo tuvo como propósito principal describir y analizar semióticamente los niveles de cooperación textual que emergen del proceso de lectura de un texto ensayístico por parte de alumnos correspondientes al ingreso 2010 de tres carreras de Licenciatura en Humanidades.

Metodológicamente, la observación se inscribe en el marco del modelo metatextual propuesto por el semiótico Umberto Eco en Lector in fabula (1981) y desarrollado en gran parte de su obra y producción intelectual, como ya se observa en Apocalípticos e integrados ante la cultura de masas (1968), Tratado de Semiótica General (1977), O bra abierta (1979) y La estructura ausente (1989).

La teoría de Eco concibe el proceso de lectura como una actividad en la que se conjugan, por una parte, un lector empírico, real, y por otra, un texto, en donde el primero puede estar o no capacitado para cooperar con unas estrategias textuales determinadas y cuya colaboración adecuada se realizará en tanto se logre (re)construir el "lector modelo" postulado en y por el texto según la intentio operis.

Desde este punto de vista, no hay lecturas “objetivas” propiamente tales, sino actualizaciones de planos de expresión (manifestación lineal del texto) siguiendo las estrategias organizacionales de un texto por parte del lector empírico.

Las vías posibles de seguir en el trayecto de actualización de un texto son flexibles, y van desde la necesidad de conocer las "circunstancias de enunciación", el conocimiento de "códigos y subcódigos” hasta el reconocimiento de "estructuras discursivas”, “narrativas”, “actanciales” e “ideológicas” mediante “extensiones parentetizadas”, “previsiones y paseos inferenciales” y confrontación de “estructuras de mundo” propias del lector empírico con las del mundo textual.

Realizados la descripción y el análisis de las distintas lecturas de los lectores empíricos seleccionados, se espera evidenciar los niveles de cooperación en relación a las estrategias textuales del contenido actualizado y, a partir de ellas, tomar conciencia de la vía y procesos asumidos en la actualización de un ensayo. 


\section{TEORÍA Y METODOLOGÍA}

En la concepción de Eco (1992), hay que distinguir entre interpretación y uso de un texto, considerando si este se utiliza como estímulo imaginativo o como texto para el goce, o bien si determinado texto considera como propio de su estrategia (y, por consiguiente, de su interpretación) la estimulación del uso más libre posible.

Puede haber, en este sentido, además de una práctica, una estética del uso libre y decodificación aberrante, que se da cuando un texto $\mathrm{O}$ ha sido escrito según un código C1 y se interpreta según un código C2 (Eco 1977: 198); sin embargo, hay que precisar si lo que se quiere es mantener activa la semiosis o interpretar un texto, pues un texto no es más que la estrategia que constituye el universo de sus interpretaciones, si no "legítimas", al menos legitimables.

En la problemática “obra abierta-obra cerrada” (Eco 1979), los textos cerrados son más resistentes al uso que los textos abiertos por estar concebidos para un Lector Modelo muy preciso, aun cuando dejen espacios de uso bastante flexibles.

Según Eco, “....un texto postula a su destinatario como condición indispensable no sólo de su propia capacidad comunicativa concreta, sino también de la propia potencialidad significativa. En otras palabras, un texto se emite para que alguien lo actualice incluso cuando no se espera (o no se desea) que ese alguien exista concreta y empíricamente" (1981: 76-77).

Sabemos también que los códigos del lector pueden diferir, total o parcialmente, de los códigos del emisor; que el código no es una entidad simple, sino, generalmente, un complejo sistema de sistemas de reglas; y que el código lingüístico no es suficiente para comprender los mensajes verbales, pues para decodificarlos se necesita, además de la competencia lingüística, una competencia circunstancial diversificada para poner en funcionamiento ciertas presuposiciones. De esta manera, se revela que una comunicación no es meramente lingüística, sino una actividad semiótica en sentido amplio, en la que varios sistemas de signos se complementan entre sí.

Esta situación se agudiza en el caso del texto escrito que un autor genera y luego entrega a una variedad de actos de interpretación. Para Eco “... el texto postula la cooperación del lector como condición de su actualización -es decir- un texto es un producto cuya suerte interpretativa debe formar parte de su propio mecanismo generativo: generar un texto significa aplicar una estrategia que incluye las previsiones de los movimientos del otro, como ocurre, por lo demás, en toda estrategia” (1981: 79).

La estrategia textual, organizada por un autor, debe referirse a una serie de competencias -más allá del conocimiento de los códigos- capaces de dar contenido a las expresiones que utiliza. Por tanto, deberá prever un Lector Modelo capaz de cooperar en la actualización textual de la forma prevista por él y de manera interpretativa, igual que él se ha movido generativamente. En este sentido, y específicamente, el lector del ensayo está llamado a desarrollar una competencia que pueda enfrentar la cita sin entrecomillado y la interrupción o quiebre forzado de ideas tradicionalmente continuas y supeditadas por jerarquía, de modo que existe la labor de compenetrarse con los procesos dialógicos que el texto establece con otras realidades.

En tal caso, el texto literario no se considera aislado de los demás hechos textuales y no textuales, sino en activa interrelación con ellos, articulando disciplinas, 
contextualizando datos, relacionando, como plantea Carrasco, “...tratando de dar sentido a elementos, situaciones y momentos históricos distintos, medios verbales y no verbales, literarios y de otras formas de discursividad” (2002: 199-210). Este hecho ha alterado también el modo de leer, mutando tanto el proceso privado referido a las expectativas particulares del lector como al rol de la teoría y metodología de interpretación (Lagos y Rojas 2012: 39-56).

Los medios a que se recurre son múltiples, entre ellos, la elección de una lengua (que excluye a quien no la habla); la elección de un tipo de enciclopedia (según la competencia intertextual o transtextual ad hoc del lector para un determinado tipo de texto); la elección de determinado patrimonio léxico y estilístico (selección de la audiencia mediante "marcas" de género discursivo, campo geográfico, etc.), entre otros. Prever un determinado Lector Modelo no significa sólo "esperar" que este exista, sino construirlo a través de los movimientos del texto.

Por tanto, lo que aquí nos interesa es la cooperación textual promovida por el texto y realizada por un lector empírico, es decir, las infinitas interpretaciones que de un texto pueden darse, y no los diferentes usos que pueda hacerse del mismo. No obstante, la interpretación se debe fijar en ciertos límites, pues la noción de interpretación supone siempre una dialéctica entre la estrategia del autor y la respuesta del lector. "Quede, pues, claro -expresa Eco- que de ahora en adelante, cada vez que se utilicen términos como Autor y Lector Modelo se entenderá siempre, en ambos casos, determinados tipos de estrategia textual” (1981: 89; las cursivas son nuestras).

Ahora bien, el lector empírico, como sujeto concreto de los actos de cooperación, debe "fabricarse" una hipótesis de Autor, deduciéndolo precisamente de los datos de la estrategia textual. La hipótesis que formula el lector empírico acerca de un Autor Modelo parece más segura -dice Eco- que la que formula el autor empírico de su Lector Modelo.

El lector empírico deduce una imagen tipo de Autor Modelo a partir de algo que precisamente se ha producido como acto de enunciación (Benveniste 1971) y que está presente textualmente como enunciado. Así, el Autor Modelo debe postular un Lector Modelo que no existe aún efectivamente y debe realizarlo como serie de operaciones textuales. En este sentido, no debe entenderse como cooperación textual la actualización de las intenciones del sujeto empírico (intentio lectoris) ni del autor empírico (intentio auctoris), sino de las intenciones que el enunciado contiene virtualmente y que el lector empírico realizará a través de ciertos niveles de cooperación textual (cfr. Lagos 2003: 31).

El modelo de Eco parte del principio de que un texto es un artificio sintácticosemántico-pragmático cuya interpretación está prevista en su propio proyecto generativo, lo cual no significa que las fases interpretativas elegidas reflejen en sentido inverso las fases generativas. Por esto, la noción de nivel textual sólo puede ser una noción teórica, un esquema metatextual que puede articularse de diversos modos en dependencia del tipo de proyecto teórico al servicio del que se encuentre, es decir, en nuestro caso, interesan los movimientos cooperativos que realiza el lector empírico de un texto escrito, especialmente de un texto narrativo artificial (en la terminología de Van Dijk, según Eco), es decir, ejemplo de descripción de acciones referidas "a unos individuos y unos hechos atribuidos a mundos posibles, distintos del de nuestra experiencia” (Eco 1981: 100).

Inspirado en el modelo generativo de Petöfi, que fija con rigidez la dirección del proceso generativo, el modelo de Eco se niega explícitamente a representar las 
direcciones y la jerarquía de las distintas fases del proceso cooperativo. El diagrama expresa el hecho de que, en el proceso concreto de interpretación, a todos los niveles y subniveles -que de hecho son "casilleros” metatextuales- también pueda llegarse dando grandes "saltos" sin tener que recorrer necesariamente casillero por casillero. En este sentido, el modelo interesa más como punto de referencia que como intento de aplicación rigurosa, permitiendo con ello modificaciones dependientes de las estrategias textuales que previenen los movimientos cooperativos del lector empírico (Figura 1).

Figura 1. Niveles de cooperación textual (Eco 1981)

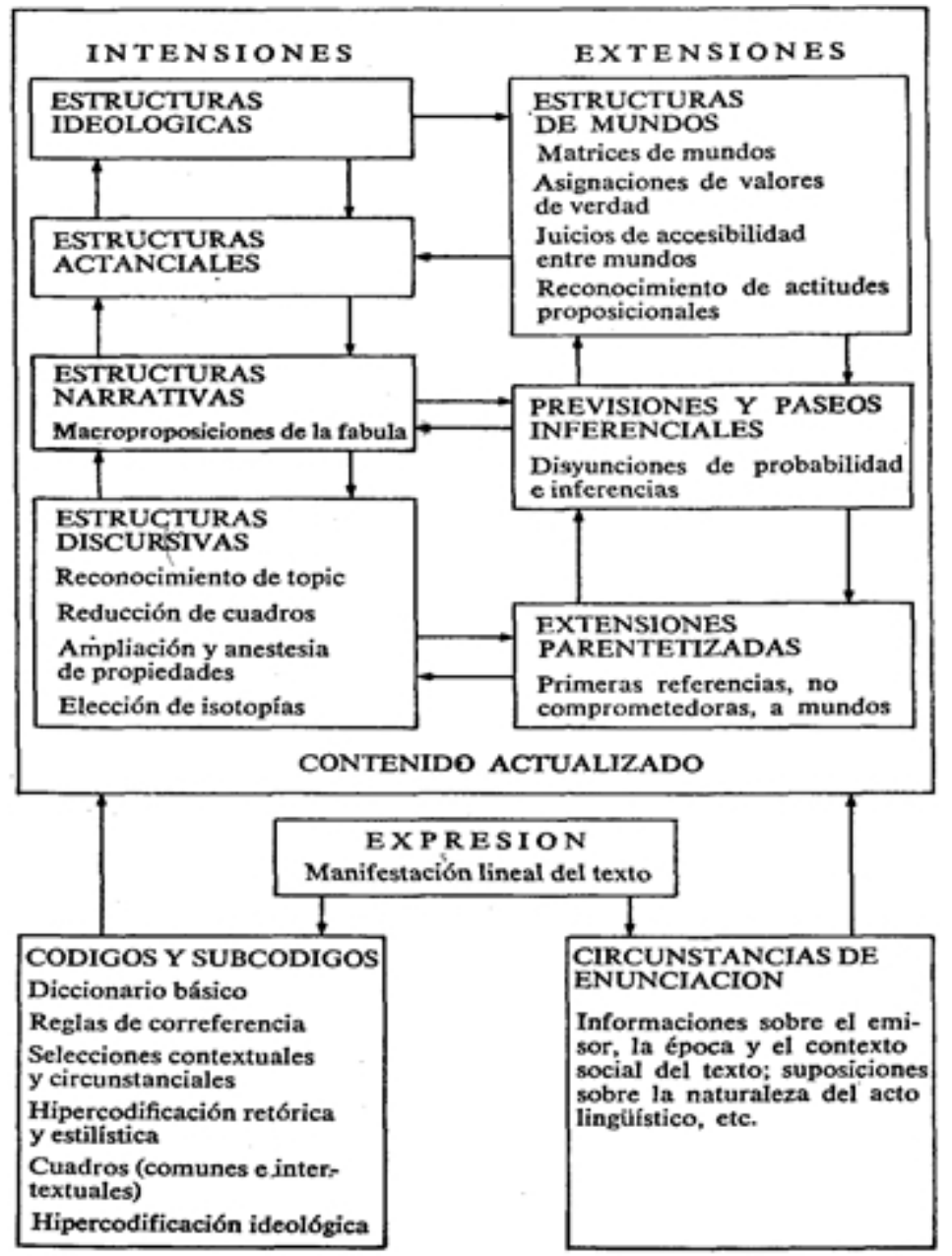

El modelo distingue básicamente dos planos sobre los cuales se realizan los movimientos cooperativos del lector empírico: el de la expresión (manifestación lineal del texto o superficie lexemática) y el del contenido actualizado. El lector empírico aplica a las expresiones determinado sistema de códigos y subcódigos para transformarlas en un primer nivel de contenido. 
Para una actualización de las estructuras discursivas (nivel de contenido) de las proposiciones narrativas escritas artificiales, el lector confronta la manifestación lineal con el sistema de códigos y subcódigos que proporciona la lengua en que el texto está escrito y la competencia enciclopédica a que esa lengua remite por tradición cultural.

Esta confrontación implica pasos cooperativos que van de las formas más simples de cooperación hasta las más complejas. Así, pues, uno de los primeros movimientos que realiza el lector empírico para poder aplicar la información que le proporcionan los códigos y subcódigos consiste en suponer, de modo transitorio, una identidad entre el mundo al que el enunciado hace referencia y el mundo de su propia experiencia, así como se refleja en el "diccionario básico".

Si en la actualización de los movimientos cooperativos se descubren discrepancias entre este mundo de la experiencia y el del enunciado (verosímil), entonces el lector deberá realizar operaciones extensionales más complejas partiendo de inferencias basadas en cuadros intertextuales, pues todo texto se lee dependientemente de la experiencia que el lector tiene de otros textos. Esta competencia intertextual (Kristeva 1974) representa una situación especial de hipercodificación y establece sus propios cuadros.

Los cuadros intertextuales son esquemas retóricos o narrativos que forman parte de un repertorio seleccionado y restringido de conocimientos que no todos los miembros de una cultura poseen. Así, algunos lectores empíricos son capaces de reconocer, por ejemplo, la transgresión de ciertas reglas de género y están en condiciones de prever más fácilmente el desenlace de una "historia”; otros, en cambio, no disponen de ciertos cuadros y están expuestos a gozar o a sufrir por las sorpresas o los hechos inusitados que les depare el enunciado (Lagos 2003: 33).

Aunque aquí se trata de ver en qué medida un texto prevé un Lector Modelo dotado de cierta competencia ideológica, también se trata de ver cómo la competencia ideológica del lector empírico (prevista o no por el texto y consciente o no de ella el lector) interviene en los procesos de actualización de los niveles de sentido de un texto en donde las actitudes ideológicas del lector pueden intervenir en la determinación del nivel de lectura.

En las previsiones y paseos inferenciales del lector empírico -actualizados los niveles discursivo y narrativo-, la cooperación remitirá permanentemente el texto a la enciclopedia; por lo tanto, el lector sale del texto para recurrir a cuadros comunes e intertextuales y confrontar los "mundos posibles" del texto con la construcción cultural de su "mundo real” de referencia.

El lector es el agente de la actualización y de la repragmatización de un texto, es el responsable de la (re)construcción del significado del texto, de su interpretación. Ante el texto, el lector actúa como un descubridor de las opciones significativas que el texto avale 0 admita.

\section{LA INVESTIGACIÓN SEMIÓTICA EMPÍRICA}

La selección de un universo de 92 informantes-estudiantes ${ }^{1}$ de ingreso 2010 a las carreras de licenciatura de la universidad tuvo como propósito, en principio, recabar

De aquí en adelante usaremos la abreviatura IE para referirnos a informantes-estudiantes. 
información y datos empíricos respecto de su performance como lectores empíricos frente a la lectura de textos seleccionados por el grupo investigador.

El equipo de investigación seleccionó varios ensayos, tanto literarios como filosóficos, de los cuales luego de ser leídos, eligió uno por considerarlo más acorde con lecturas habituales y próximas de alumnos de primer año universitario.

El texto elegido, "El exilio y la literatura”, un discurso pronunciado en Viena por el escritor Roberto Bolaño (cf. infra), fue aplicado tres veces a tres grupos de control de alumnos de primeros años de distintas carreras. Los resultados condujeron a la reelaboración del instrumento por considerar que las preguntas dieron lugar a respuestas direccionadas que no coincidían con los propósitos del trabajo ni con el modelo semiótico que permitía la observación.

El instrumento fue reelaborado con sólo dos instrucciones, incluidos datosantecedentes de los IE, que dieron lugar a respuestas abiertas:

1. Haga un resumen del texto leído

2. Haga un comentario del texto leído.

El instrumento aplicado y sus resultados, luego fueron trasladados a una tabla de apreciación en la cual se categorizan las diferentes lecturas en parámetros de acuerdo a la mayor o menor cooperación en la construcción del lector modelo propuesto en el texto. Este instrumento de análisis de los datos consiste en una escala de apreciación en donde están contenidos los diferentes niveles de cooperación textual que los lectores deben generar en el proceso lector. Estos niveles de cooperación textual, a su vez, están subdivididos de modo que el instrumento pueda adecuarse al análisis de las lecturas de los informantes-estudiantes.

Los grados de apreciación del instrumento van de 1 a 5, en donde cada nivel está compuesto por una descripción apreciativa consistente con el indicador de logro. De esta manera, el instrumento en total posee un máximo de 120 y un mínimo de 24 puntos. Entre estos parámetros se clasificaron los movimientos cooperativos de los estudiantes de acuerdo a la mayor o menor cooperación que se logra con el texto en términos de la construcción del lector modelo propuesto en el ensayo.

Las respuestas de los IE fueron examinadas teniendo como referencia el esquema metatextual de Eco y la lectura “modelo” del ensayo de Bolaño realizada por el grupo investigador y ampliado por Lagos y Rojas (cfr. 2012) en conjunción con los rasgos fundamentales del género ensayístico.

\section{Resultados}

En primer lugar, precisemos que el universo de 92 IE se constituyó de 13 IE de Licenciatura en Lenguaje y Comunicación, 36 IE de Licenciatura en Historia y Geografía y 43 IE de Licenciatura en Inglés. De este universo 85 IE completaron el instrumento y 7 se abstuvieron, aunque permanecieron en la sala el tiempo asignado.

La descripción y el análisis de las distintas lecturas de los lectores empíricos seleccionados hicieron posible determinar las estrategias textuales y las vías posibles de seguir en el trayecto de actualización y con ello la identificación de los niveles de cooperación inadecuados y adecuados emergentes del proceso de lectura.

En una vista general de los resultados de la aplicación del instrumento, fue posible determinar que el mayor porcentaje de IE -56,5 \%- (48 IE) son categorizados 
como lectores que cooperan con el texto, no obstante, omiten información relevante produciéndose algunas deficiencias que hacen difícil la construcción del lector modelo. Estas deficiencias se evidencian en la carencia del reconocimiento e identificación de aspectos ideológicos presentes en el texto, tales como la propuesta del nuevo concepto de exilio, entre otros. La información omitida corresponde esencialmente a la referida a la generación de paseos inferenciales adecuados respecto al contenido del texto. El ensayo "El exilio y la literatura” presenta una serie de elementos que exigen que el lector active constantemente su enciclopedia y conocimiento de mundo.

Por otra parte, el $40 \%$ de los IE (34) de la muestra se categoriza como lectores que cooperan adecuadamente con el texto, no obstante, omiten algunos elementos de comentarios relevantes que coincidentemente corresponden a la generación de paseos inferenciales que los lectores silencian y el $2 \%$ de los IE (2) no coopera con el texto, por lo que su lectura es abiertamente deficiente.

Cabe destacar que solo 1 de los IE permite la construcción del lector propuesto por la lectura por cuanto se ubica en el tramo de logro más alto con 100 puntos de un máximo de 120. Este IE coopera con el texto de manera adecuada, esto es, lo actualiza y repragmatiza, asumiendo la responsabilidad de (re)construir su significado e interpretarlo. Ante el texto, solo este lector actúa como un descubridor de las opciones significativas que avala o admite. Del mismo modo, atiende las condicionantes y la subjetividad del texto, ante lo cual hace uso de sus saberes para apropiarse del contenido del mensaje e interactuar con él. Pese a no haber alcanzado el máximo puntaje en el desarrollo de las dos actividades solicitadas, evidencia que al llevar a cabo la lectura apela a su experiencia y colabora con las vías que el texto sugiere, aplicando las estrategias de comprensión e interpretación y las pautas metacognitivas adecuadas. Se entiende que este lector está dotado de específicos conocimientos previos y experiencias lectoras que le permiten identificar e interpretar legítimamente las referencias textuales a través de una activa cooperación de su intertexto en la que intervienen las ineludibles aportaciones de las variables personales.

Lo anterior se puede observar en la siguiente categorización realizada a partir de las actualizaciones de los 85 IE (Cuadro 1):

Cuadro 1

\begin{tabular}{|c|c|c|}
\hline Parámetro de puntajes & DESCRIPCIÓN & N I.E. $^{\circ}$ \\
\hline Entre 101 y 125 puntos & $\begin{array}{c}\text { El estudiante coopera con el texto de manera adecuada } \\
\text { construyendo el “lector modelo" propuesto en la lectura. }\end{array}$ & 34 \\
\hline Entre 71 y 100 puntos & $\begin{array}{c}\text { El estudiante coopera adecuadamente con el texto, sin embargo, } \\
\text { omite algunos elementos de comentario relevantes. }\end{array}$ & 48 \\
\hline Entre 31 y 70 puntos & $\begin{array}{c}\text { El estudiante coopera con el texto, no obstante, la omisión de } \\
\text { elementos relevantes de comentario produce algunas deficiencias en } \\
\text { la lectura. }\end{array}$ & $\begin{array}{c}\text { El estudiante no coopera con el texto. Su lectura es abiertamente } \\
\text { deficiente. }\end{array}$ \\
\hline 30 puntos y menos & & 2 \\
\hline
\end{tabular}

En un análisis más específico de los resultados obtenidos de la aplicación del instrumento a los IE en cada una de las carreras, es posible observar que los porcentajes alcanzados en los Niveles de Cooperación del Modelo Textual de Eco son los siguientes (Cuadro 2): 
Cuadro 2

\begin{tabular}{|l|c|c|c|}
\hline \multicolumn{1}{|c|}{$\begin{array}{c}\text { Niveles de Cooperación } \\
\text { Textual }\end{array}$} & Licenciatura 1 & Licenciatura 2 & Licenciatura 3 \\
\hline N 1 Estructuras de mundo & $79 \%$ & $64,3 \%$ & $58,7 \%$ \\
\hline N 2 Estructuras narrativas & $76,3 \%$ & $60,4 \%$ & $54,3 \%$ \\
\hline N 3 Previsiones y paseos inferenciales & $61,5 \%$ & $30,9 \%$ & $45,1 \%$ \\
\hline N 4 Circunstancias de enunciación & $73,4 \%$ & $46 \%$ & $54,2 \%$ \\
\hline N 5 Estructuras actanciales e ideológicas & $77,8 \%$ & $43,6 \%$ & $46,9 \%$ \\
\hline
\end{tabular}

En el Cuadro 2 se evidencia que el trayecto con mayor frecuencia es el que realiza la totalidad de los IE en el Nivel Estructuras de Mundo, por cuanto, las tres carreras presentan el más alto porcentaje del puntaje asignado a las variables que lo componen, según la Escala de Apreciación.

Por otra parte, el trayecto de menor frecuencia es el que sigue la totalidad de los IE en el Nivel Previsiones y Paseos Inferenciales, por cuanto nuevamente son las tres carreras las que presentan el porcentaje más bajo del puntaje asignado. Pese a que 34 IE de los 85 cooperan con el texto, no logran realizar previsiones o paseos inferenciales, omitiendo con ello elementos relevantes de análisis que produce confusiones en la interpretación de la actualización.

En un análisis globalizador y considerando solo a los 85 IE que desarrollaron el instrumento, se puede apreciar, respecto de la identificación de cada una de las estructuras metatextuales referidas en el texto, los resultados de la siguiente manera (Cuadro 3):

Cuadro 3

\begin{tabular}{|l|c|c|c|c|}
\hline \multirow{2}{*}{ ESTRUCTURAS/CATEGORÍA } & \multicolumn{2}{c|}{$\begin{array}{c}\text { Identifica estructura en la } \\
\text { lectura }\end{array}$} & \multicolumn{2}{c|}{ No identifica estructura en la lectura } \\
\cline { 2 - 5 } & $\mathrm{N}^{\circ} \mathrm{IE}$ & $\%$ & $\mathrm{~N}^{\circ} \mathrm{IE}$ & $\%$ \\
\hline Estructuras de mundo & 19 & 22,3 & 66 & 77,7 \\
\hline Estructuras narrativas & 17 & 20 & 83 & 80 \\
\hline Estructuras actanciales e ideológicas & 17 & 20 & 83 & 80 \\
\hline Paseos inferenciales & 6 & 7,1 & 79 & 92,9 \\
\hline Circunstancias de enunciación & 12 & 14,1 & 73 & 85,9 \\
\hline
\end{tabular}

Una de las primeras aproximaciones en la aplicación de un enfoque semiótico al problema de la comprensión lectora, arroja que la comprensión del texto está estrechamente sujeta a la estructura organizacional textual de acuerdo a la intentio operis.

Los movimientos cooperativos que un lector empírico realiza en el proceso lector configuran de algún modo las posibles interpretaciones que puedan concluirse. Asimismo, la forma del texto predispone al lector a generar diversas lecturas y aproximarse al contenido de una manera específica.

En esta investigación se ha analizado un texto ensayístico, por lo tanto el lector debe enfrentarse a él con plena conciencia del tipo de texto que posee frente a él. Sin embargo, la enseñanza de la comprensión lectora carece de reconocimiento de 
hipercodificaciones, pues no se enfatiza la identificación de las diversas modalidades discursivas o tipologías textuales como punto inicial en el proceso lector.

Cuando un lector empírico no conoce la forma del recorrido de la lectura y no conoce su hipercodificación, difícilmente podrá configurar su lector modelo mediante las estrategias textuales presentadas en el texto. Es un caminar a tientas, a ciegas. En este sentido, la perspectiva semiótica propuesta en esta investigación para abordar la problemática de la comprensión lectora proporciona al análisis mayores elementos de entendimiento de los diferentes procesos que ocurren en la lectura.

Los diferentes movimientos cooperativos que realizan los lectores empíricos al momento de enfrentarse a un texto evidencian también los procesos internos que el lector genera en la lectura. Por lo tanto, un enfoque semiótico da las respuestas necesarias a estos movimientos cooperativos de generar un mejor entendimiento del proceso lector.

El menor recorrido de lectura realizado por los IE dice relación con el noreconocimiento de las previsiones y paseos inferenciales y las circunstancias de enunciación. Muy pocos estudiantes realizan paseos inferenciales relevantes para la configuración del lector modelo. Es más, una vez analizadas las respuestas dadas por los estudiantes a partir de sus lecturas, se puede afirmar que los paseos inferenciales realizados por los estudiantes son abiertamente deficientes, es decir, pocos estudiantes son capaces de relacionar el contenido del texto con las experiencias de mundo y el conocimiento previo. Respecto a las circunstancias de enunciación, también son pocos los estudiantes que identifican esta estructura y la hacen relevante en el proceso de lectura.

Finalmente, se pueden sintetizar los resultados de esta investigación del siguiente modo (Cuadro 4):

Cuadro 4

\begin{tabular}{|l|c|}
\hline \multicolumn{2}{|c|}{ Configuración del lector modelo a partir del recorrido metatextual } \\
\hline Lectura óptima próxima a la elaboración del Lector Modelo & 35 estudiantes/41 \% \\
\hline Lectura deficiente & 50 estudiantes/59 \% \\
\hline T O T A L & 85 estudiantes $/ 100 \%$ \\
\hline
\end{tabular}

\section{CONCLUSIONES}

Esta investigación se presenta como la operacionalización de una metodología de análisis semiótico metatextual de la lectura.

La descripción de los distintos niveles que emergieron de la lectura del ensayo realizada por los estudiantes evaluados dio como resultado un recorrido metatextual por las diferentes estructuras presentadas en el modelo de Umberto Eco. De esta forma, esta investigación constituye un precedente en cuanto a la investigación en comprensión lectora, puesto que propone una nueva mirada para este fenómeno.

Los estudios lingüísticos han hecho grandes aportes teóricos y metodológicos en este campo; no obstante, la combinación de estas dos miradas, la semiótica y la lingüística, arroja como resultado una nueva perspectiva del fenómeno lector considerando los procesos metacognitivos y textuales propiamente tales. 
Leer es un proceso colaborativo, constructivo, integrador, estratégico y metacognitivo. Es colaborativo porque el lector va construyendo mentalmente una propuesta personal respecto al significado del texto. Es obvio que el lector no permanece pasivo frente a la lectura; sino que él es conducido por el texto en la búsqueda de significados. Esto es una constante, porque en la medida de su avance, irá formulando hipótesis de lecturas las que se validarán o no en el texto. La lectura también es un proceso integrador, en la medida en que se fusionan los conocimientos previos con la información nueva proporcionada por el texto.

No tan solo es un problema, entonces, que el vocabulario no sea conocido, sino que haya falta de recursos enciclopédicos para deducir los significados, pues si estos se dan, la lectura se hará más fluida, grata y reconfortante. Asimismo, leer es un proceso estratégico, porque supone una actitud lectora, de acuerdo al tipo de lectura y al interés que se tenga sobre ella.

Esto exige un lector flexible, pues no se sujetará a un solo tipo de lectura, sino que optará por la estrategia pertinente según cuál sea el reto a enfrentar. Esto implica que el lector tiene algún objetivo al leer un texto motivado extrínseca o intrínsecamente por las estrategias del texto.

Finalmente, el proceso de lectura debe enfrentar una primera etapa metacognitiva, puesto que el lector debe ser consciente de cómo lee, qué caminos sigue para comprender y si realmente está leyendo orientado por las estrategias del texto. Esto es, si maneja su lectura y puede tomar las acciones correctivas necesarias. Aquí el lector aprecia cuánto le son útiles las marcas en la lectura, el parafraseo, los procesadores de información (esquemas, diagramas, etc.) y los organizadores visuales.

En este sentido, esta perspectiva semiótica proporciona las herramientas teóricometodológicas para una lectura metacognitiva. Los procesos mentales que ocurren en el lector en la medida que actualiza la estructura y el contenido del texto, son descritos en el modelo de Eco. No obstante, y en último término, se trata de que el mismo lector sea capaz de analizar su propio proceso lector y de esa manera obtener mejores resultados en la comprensión global de un texto. Las diferentes estrategias metacognitivas utilizadas por los lectores y la conciencia de ellas dependerán en gran medida de la experiencia lectora de los mismos.

Así, un lector consciente de su proceso será mucho más competente que un lector ingenuo. Pero no es solo esto. La destreza de la comprensión lectora radica en gran medida en el desarrollo de esta competencia semiótica.

La competencia lingüística, es decir, el manejo de un código lingüístico específico no asegura en gran medida la comprensión del significado textual. Es preciso premunirse de una enciclopedia adecuada y pertinente al tipo de texto al que el lector se enfrenta. A esto le denominamos competencia semiótica. Y es la combinación de estas dos competencias la que asegura la comprensión lectora en el proceso de (re) construcción del lector modelo postulado por todo texto.

Esta investigación empírica dejó de manifiesto que la competencia semiótica es gravitante en el fenómeno de la comprensión lectora. Lograr la correcta actualización del contenido textual y finalmente (re)construir su lector modelo no es simplemente un proceso de decodificación lingüística, sino que, además de ello, consiste en una progresión por el contenido textual a través de las diferentes estructuras metatextuales. La intentio operis guía al lector por diferentes caminos quien a su vez deberá recorrerlos de acuerdo a las mismas estrategias que el texto ofrece para su lectura. De 
este modo, un lector avezado realizará este recorrido con tal conciencia y ejecutando diversas estrategias metacognitivas que le permitan alcanzar la comprensión del significado textual, y, por supuesto, caer también en la cuenta cuando un texto esté organizativamente mal construido o, intencionalmente, incluya "trampas narrativas" (y sea un éxito metatextual), como Un drame bien parisien de Alfonso Allais, texto trabajado como experiencia empírica por Eco, primero, con sus alumnos del Instituto di Discipline della Comunicazione e dello Spettacolo de la Universidad de Bolonia y luego en el Centro Internazionale di Semiotica e Linguistica de la Universidad de Urbino (cfr. Eco 1981: 274-306).

Un último elemento que debe considerarse en la comprensión textual desde una perspectiva semiótica es la hipercodificación, como ya lo mencionáramos (v. supra), es decir, el reconocimiento sociocultural -no siempre coincidente con la comunidad científica ad hoc, del formato textual o modalidad discursiva a la que el lector se enfrenta.

Sabido es que el formato textual condiciona en gran medida el proceso de lectura e interpretación del contenido del texto. En este sentido, este trabajo investigativo evidenció que muchos de los IE no reconocieron el tipo textual al que se enfrentaron y por lo tanto su lectura resultó dispersa y azarosa. Las distintas tipologías discursivas mueven al lector hacia caminos de cooperación diferentes. No será lo mismo, por ejemplo, leer un texto argumentativo u otros que leer un texto de intención literaria. Por lo tanto, el conocimiento enciclopédico del lector no sólo abarca en parte el saber de qué trata el texto, sino también, y con antelación, saber qué tipo de texto se está leyendo.

\section{OBRAS CITADAS}

Baumann, James. 2001. La comprensión lectora. Madrid: Machado Libros.

Benveniste, Émile. 1971. Problemas de Lingüística general. Buenos Aires: Siglo XXI

Cairney, Trevor. 1992. Enseñanza de la comprensión lectora. Madrid: Morata.

Carrasco, Iván. 2002. "Interdisciplinariedad, interculturalidad y canon en la poesía chilena e hispanoamericana actual”. Estudios Filológicos 37: 199-210

Cassany, Daniel. 2006. Entre líneas: Sobre la lectura contemporánea. Barcelona: Anagrama.

Eco, Umberto. 1968. A pocalípticos e integrados ante la cultura de masas. Barcelona: Lumen. 1977. Tratado de semiótica general. Barcelona: Lumen

1979. O bra abierta. Barcelona: Ariel.

.1981. Lector in fabula. Barcelona: Lumen.

1989. La estructura ausente. Barcelona: Lumen.

. 1992. Los límites de la interpretación. Barcelona: Lumen.

Greimas, André. J. 1993. La semiótica del texto. Barcelona: Paidós.

Halliday, Michael. 1986. El lenguaje como semiótica social. México: F.C.E.

Kristeva, Julia. 1974. El texto de la novela. Barcelona: Lumen.

Lagos, Jorge. 1992. "Proposición teórico-metodológica para un estudio empírico sobre estética de la recepción. Informe de un caso.” Estudios Filológicos 27: 131- 139

. 1998. "Códigos y variantes de código estéticos en estudiantes de Enseñanza Media.” Estudios Filológicos 33: 155-167

. 2003. La metalepsis y la actividad cooperativa del lector empírico. Valdivia: Estudios

Filológicos Anejo 16.

Lagos, Jorge y Rojas, Daniel. 2012. "Proceso de actualización de un ensayo de Roberto Bolaño”. Estudios F ilológicos 50: 39-56 
Peronard, Marianne; Gómez, Luis; Parodi, Giovanni y Núñez Paulina. 1997. Comprensión de textos escritos: de la teoría a la sala de clases. Santiago de Chile: Andrés Bello.

Peronard, Marianne. 2005. "La metacognición como herramienta didáctica”. Revista Signos 57: 61-74 . 2007. "Lectura en papel y en pantalla de computador. Revista Signos 63: 179-195.

Solé, Isabel. 1992. Estrategias de lectura. Barcelona: Graó.

Van Dijk, Teun. 1995. Texto y Contexto. Madrid: Cátedra. 1996. La ciencia del texto. $4^{a}$ ed. Barcelona: Paidós.

2001. "Algunos principios de la teoría del contexto." Revista Latinoamericana de Estudios del Discurso 1, 1: 69-81. 